\title{
Meridional Circulation and Global Solar Oscillations
}

\author{
M. Roth • M. Stix
}

Received: 5 October 2007 / Accepted: 11 June 2008 / Published online: 23 July 2008

(C) The Author(s) 2008. This article is published with open access at Springerlink.com

\begin{abstract}
We investigate the influence of large-scale meridional circulation on solar $p$ modes by quasi-degenerate perturbation theory, as proposed by Lavely and Ritzwoller (Roy. Soc. Lond. Phil. Trans. Ser. A 339, 431, 1992). As an input flow we use various models of stationary meridional circulation obeying the continuity equation. This flow perturbs the eigenmodes of an equilibrium model of the Sun. We derive the signatures of the meridional circulation in the frequency multiplets of solar $p$ modes. In most cases the meridional circulation leads to negative average frequency shifts of the multiplets. Further possibly observable effects are briefly discussed.
\end{abstract}

Keywords Helioseismology, direct modeling · Interior, convection zone · Oscillations, solar · Velocity fields, interior · Waves, acoustic, modes

\section{Introduction}

Meridional circulation is a large-scale flow observed on both hemispheres of the solar surface (Duvall, 1979; Hathaway, 1996; Komm, Howard, and Harvey, 1993). Its predominant direction is from the Equator to the Poles, and its amplitude is of the order of $15 \mathrm{~m} \mathrm{~s}^{-1}$. As mass does not accumulate in the polar regions, a return flow from the Poles to the Equator is suspected deeper within the solar interior. The top half of the convection zone contains approximately $0.25 \%$ of the solar mass, the mass of the bottom half is approximately five times larger. Consequently, a poleward flow of $10 \mathrm{~m} \mathrm{~s}^{-1}$ in the top half of the convection zone

Helioseismology, Asteroseismology, and MHD Connections

Guest Editors: Laurent Gizon and Paul Cally.

M. Roth ( $₫)$

Max-Planck-Institut für Sonnensystemforschung, Katlenburg-Lindau, Germany

e-mail: roth@mps.mpg.de

M. Stix

Kiepenheuer-Institut für Sonnenphysik, Freiburg, Germany

e-mail: stix@kis.uni-freiburg.de 
could be compensated by an equatorward flow of $2 \mathrm{~m} \mathrm{~s}^{-1}$ in the lower half. The transport of magnetic flux from mid to low latitudes by such a flow at the bottom of the convection zone would last approximately ten years, which is close to the period of the solar magnetic cycle.

In addition to magnetic flux, the meridional flow also transports angular momentum. Indeed, the circulation played a key role in early theories of the solar non-uniform rotation as well as of the magnetic cycle (Bjerknes, 1926; Kippenhahn, 1963). More recently, differential rotation has been explained in mean-field models as a consequence of the Reynolds stresses (Rüdiger, 1980; Küker and Stix, 2001; Küker and Rüdiger, 2005), and in threedimensional numerical models by the influence of the Coriolis force on global convection (Miesch et al., 2000; Miesch, Brun, and Toomre, 2006). Nevertheless, meridional circulation occurs as well in these models, and in solar-cycle models it has regained popularity, since the mean-field latitude migration along the surfaces of isorotation that occurs in the traditional $\alpha \Omega$ dynamo (Parker, 1955) does not seem to suffice. The effect of the circulation on the butterfly diagram had been demonstrated by Roberts and Stix (1972); it is considered to be essential in more recent versions of the $\alpha \Omega$ dynamo, which therefore have been termed "flux-transport dynamos" (Choudhuri, Schüssler, and Dikpati, 1995; Dikpati and Charbonneau, 1999; Nandy and Choudhuri, 2002; Rempel, 2006a, 2006b).

Local helioseismology has investigated the strength of the meridional flow in the solar interior. By means of ring-diagram analysis (Hill, 1988), Haber et al. (2002) inverted data for the circulation in a $15 \mathrm{Mm}$ deep region below the solar surface. In data from 1998 they found a flow emerging at high northern latitudes with equatorward orientation. This was interpreted as an evolving second cell of circulation. Further studies on the evolution of the flow either by ring-diagrams and by time-distance helioseismology (e.g., Zhao and Kosovichev, 2004; Zaatri et al., 2006) show predominantly a poleward flow with a strong variability in the outer $15 \mathrm{Mm}$ of the Sun. The velocity reaches $40 \mathrm{~m} \mathrm{~s}^{-1}$. These findings were interpreted as the upper parts of meridional circulation cells in the two hemispheres.

Theoretically, the influence of global-scale stationary flows on solar $p$ modes was studied in detail by quasi-degenerate perturbation theory (Lavely and Ritzwoller, 1992). These studies were successfully used to solve the forward problem for the influence of differential rotation on the $p$ modes. The results lead to an improved inversion method for determining the radial dependence of the differential rotation (Ritzwoller and Lavely, 1991). Following Lavely and Ritzwoller (1992), Roth and Stix (1999) studied the influence of large-scale sectoral poloidal flow components that could be related to giant convection cells. They found that such flows yield additional frequency shifts that can only be described with quasidegenerate perturbation theory, as these frequency shifts are effects of higher order. In a subsequent study Roth, Howe, and Komm (2002) were able to show that sectoral poloidal flows could only be found with the current inversion methods of global helioseismology as long as they exceed an amplitude of $10 \mathrm{~m} \mathrm{~s}^{-1}$. The meridional flow was found to be not detectable by the current inversion methods as the frequency splittings are fitted by an incomplete set of basis functions, which are tailored to measure only zonal toroidal flows. However, no detailed study on the effect of the meridional circulation on the oscillation frequencies was given, and few other attempts to derive observable signatures of the meridional flow in global helioseismology data exist (Woodard, 2000).

In this contribution we concentrate on a theoretical study of the influence of the meridional circulation on the solar $p$-mode frequencies and describe a possibly observable effect. This effect is significantly smaller than the frequency splitting caused by solar differential rotation. But as time series of global oscillation data exist that cover more than ten years, the necessary frequency resolution might be available to detect it. The advantage of studying the meridional circulation by global helioseismic techniques is a possible inference of information from greater depths. 


\section{Frequency Shifts Caused by Meridional Circulation}

The effect of the meridional circulation on solar oscillations shall be investigated by solving the forward problem. We use quasi-degenerate perturbation theory as proposed by Lavely and Ritzwoller (1992) to calculate shifts of the oscillation frequencies. A short outline of the mathematics is given in the following.

As described by Lavely and Ritzwoller (1992) and Roth and Stix (1999), we consider a spherically symmetric equilibrium model of the Sun. For this purpose "Model S" from Christensen-Dalsgaard et al. (1996) is used. The solar $p$ modes are adiabatic eigenoscillations $\left(\boldsymbol{\xi}_{k}\right)$ of small amplitude, where $k$ stands for the three indices: harmonic degree $(\ell)$, azimuthal order $(m)$, and radial order $(n)$. We calculate the eigenfrequencies and eigenmodes numerically with the http://www.phys.au.dk/ jcd/adipack.n/ ADIPACK code (ChristensenDalsgaard and Berthomieu, 1991).

The equation governing the eigenmodes is

$$
\mathcal{L}_{0} \boldsymbol{\xi}_{k}=-\rho_{0} \omega_{k}^{2} \boldsymbol{\xi}_{k}
$$

where $\rho_{0}$ is the density and $\omega_{k}$ the oscillation frequency. The operator $\mathcal{L}_{0}$ acts on the eigenoscillation by

$$
\mathcal{L}_{0} \boldsymbol{\xi}=-\nabla P^{\prime}+\rho_{0} \boldsymbol{g}^{\prime}+\rho^{\prime} \boldsymbol{g}_{0},
$$

where the primed quantities are the Eulerian variations of pressure, gravitational acceleration, and density caused by an oscillation mode (e.g., Stix, 2004; Unno et al., 1989).

Describing the disturbing effect of the meridional circulation, we replace in Equations (1) the operator $\mathcal{L}_{0}$, the squared eigenfrequency $\omega_{k}^{2}$, and the eigenfunction $\boldsymbol{\xi}_{k}$ with

$$
\begin{aligned}
\mathcal{L}_{0} & \rightarrow \mathcal{L}_{0}+\mathcal{L}_{1}, \\
\omega_{k}^{2} & \rightarrow \tilde{\omega}_{j}^{2}, \\
\boldsymbol{\xi}_{k} & \rightarrow \tilde{\boldsymbol{\xi}}_{j},
\end{aligned}
$$

where the perturbation operator $\mathcal{L}_{1}$ is defined in terms of the meridional circulation $\boldsymbol{u}_{0}$ and acts on the eigenmodes as

$$
\mathcal{L}_{1}\left(\boldsymbol{\xi}_{k}\right)=-2 \mathrm{i} \omega_{\text {ref }} \rho_{0}\left(\boldsymbol{u}_{0} \cdot \nabla\right) \boldsymbol{\xi}_{k}
$$

The perturbed eigenfunctions are expressed in terms of the unperturbed normal modes of the equilibrium model

$$
\tilde{\boldsymbol{\xi}}_{j}=\sum_{k \in \mathcal{K}} a_{k}^{j} \boldsymbol{\xi}_{k},
$$

where the index $k$ and the subscript $j$ are elements of the subspace $\mathcal{K}$ that is spanned by the eigenfunctions which are quasi-degenerate. We identify $j$ with that $k$ for which the expansion coefficients $a_{k}^{j}$ are maximal in magnitude. Following Lavely and Ritzwoller (1992), we use

$$
\tilde{\omega}^{2}=\omega_{\text {ref }}^{2}+\lambda
$$

for the perturbed frequency. The reference frequency $\left(\omega_{\text {ref }}\right)$ should be chosen in the vicinity of the eigenfrequencies of the modes in the subspace $\mathcal{K}$. 
According to Lavely and Ritzwoller (1992), the problem can be turned into an algebraic eigenvalue problem by substituting Equations (5) and (6) into the perturbed eigenvalue problem and making use of the orthogonality of the eigenfunctions

$$
\sum_{k \in \mathcal{K}} a_{k}^{j} Z_{k^{\prime} k}=\sum_{k \in \mathcal{K}} a_{k}^{j} \lambda_{j} \delta_{k^{\prime} k} \quad \text { for } k^{\prime} \in \mathcal{K}
$$

The elements of the matrix $\mathbf{Z}$ are given by

$$
Z_{k^{\prime} k}=\frac{1}{N_{k^{\prime}}}\left\{H_{n^{\prime} n, \ell^{\prime} \ell}^{m^{\prime} m}-\left(\omega_{\text {ref }}^{2}-\omega_{k}^{2}\right) N_{k} \delta_{k^{\prime} k}\right\},
$$

with

$$
H_{n^{\prime} n, \ell^{\prime} \ell}^{m^{\prime} m}=-\int \xi_{k^{\prime}}^{*} \cdot \mathcal{L}_{1} \xi_{k} \mathrm{~d}^{3} r
$$

The normalization of the eigenfunctions is given by

$$
N_{k}=\int_{0}^{R_{\odot}} \rho_{0}\left[\xi_{r}^{2}+\ell(\ell+1) \xi_{h}^{2}\right] r^{2} \mathrm{~d} r .
$$

\subsection{The Model of the Meridional Circulation}

We use a linear superposition of Legendre polynomials $\left[P_{s}(\cos \theta)\right]$ with degree $s$ for our representation for the meridional velocity field

$$
\boldsymbol{u}_{0}=\sum_{s} u_{s}(r) c_{s} P_{s}(\cos \theta) \boldsymbol{e}_{r}+v_{s}(r) \nabla_{\mathrm{h}} c_{s} P_{s}(\cos \theta)
$$

where $\nabla_{\mathrm{h}}$ is a horizontal gradient and $\theta$ is the colatitude. The factor $c_{s}$ is used for normalization of the Legendre polynomials and is determined by $c_{s}^{2}=(2 s+1) / 4 \pi$. The expansion coefficients $u_{s}(r)$ and $v_{s}(r)$ are functions of the radial coordinate $(r)$, and represent the radial and horizontal strength of the flow component with degree $s$. In this model the meridional velocity field is a zonal poloidal flow which is independent of longitude.

The meridional circulation shall be free of divergence, i.e., $\nabla \cdot\left(\rho_{0} \boldsymbol{u}_{0}\right)=0$, which enables us to express $v_{s}$ in terms of $u_{s}$,

$$
\rho_{0} r s(s+1) v_{s}=\partial_{r}\left(r^{2} \rho_{0} u_{s}\right)
$$

We adopt a simple model for the depth dependence of $u_{s}(r)$, which is given by

$$
\begin{aligned}
& u_{s}(r)=A \sin \left(n_{\mathrm{c}} \pi \frac{r-r_{\mathrm{b}}}{R_{\odot}-r_{\mathrm{b}}}\right) \quad \text { for } r_{\mathrm{b}} \leq r \leq R_{\odot}, \\
& u_{s}(r)=0 \text { otherwise. }
\end{aligned}
$$

The return flow of the meridional flow is closed at the radius given by the parameter $r_{b}$, which we set equal to the bottom of the convection zone $r_{\mathrm{b}}=0.713 R_{\odot}$ (Basu and Antia, 1997). The number of circulation cells in depth is defined by the parameter $n_{\mathrm{c}}$. We select the amplitude $(A)$ such that the horizontal flow component $\left(v_{s}\right)$ at the solar surface has a maximum amplitude of $15 \mathrm{~m} \mathrm{~s}^{-1}$. 
Below we present results of the effects on the solar $p$-mode frequencies of 15 various models of the meridional circulation. We vary $s$ between two and six and $n_{\mathrm{c}}$ between one and three. The higher degrees $s$ represent more than one cell in each hemisphere; such multi-cell circulation occurs in numerical simulations (Brun and Toomre, 2002; Miesch, Brun, and Toomre, 2006) and has been observed by means of sunspot tracing on the solar surface (Tuominen, Tuominen, and Kyröläinen, 1983; Wöhl and Brajša, 2001). The models with odd $s$ have an equator-crossing flow; we include these as such asymmetric flows have been found on the Sun as well (Haber et al., 2000; Zhao and Kosovichev, 2004). Figure 1 illustrates the 15 models.

Due to Equation (12), models with higher degree $s$ show stronger radial flows in the down- and upflow channels whereas the horizontal flow strength is the same in all models.

\subsection{Theoretical Frequency Shifts}

We evaluate the integration of Equation (9) by using the model of the meridional circulation (11) and (12). The integral over longitude does not vanish only if $m^{\prime}=m$. The result is

$$
\begin{aligned}
H_{n^{\prime} n, \ell^{\prime} \ell}(m)= & 8 \mathrm{i} \omega_{\text {ref }} \pi(-1)^{m} \gamma_{\ell} \gamma_{\ell^{\prime}} \\
& \times \sum_{s} \gamma_{s} \int_{0}^{R_{\odot}} u_{s}\left[R_{s}-\partial_{r}\left(\frac{H_{s}}{s(s+1)}\right)\right] \rho_{0} r^{2} \mathrm{~d} r\left(\begin{array}{ccc}
s & \ell & \ell^{\prime} \\
0 & m & -m
\end{array}\right) .
\end{aligned}
$$

The integral kernels $R_{s}(r)$ and $H_{s}(r)$ are the equivalents of the more general poloidal-flow kernels given in Lavely and Ritzwoller (1992)

$$
\begin{aligned}
R_{s}(r)= & \frac{1}{4}\left(\xi_{r}^{\prime} \frac{\partial \xi_{r}}{\partial r}-\frac{\partial \xi_{r}^{\prime}}{\partial r} \xi_{r}\right)\left(1+(-1)^{s+\ell+\ell^{\prime}}\right)\left(\begin{array}{ccc}
s & \ell & \ell^{\prime} \\
0 & 0 & 0
\end{array}\right) \\
& +\frac{1}{2}\left(\xi_{h}^{\prime} \frac{\partial \xi_{h}}{\partial r}-\frac{\partial \xi_{h}^{\prime}}{\partial r} \xi_{h}\right)\left(1+(-1)^{s+\ell+\ell^{\prime}}\right) \Omega_{\ell} \Omega_{\ell^{\prime}}\left(\begin{array}{ccc}
s & \ell & \ell^{\prime} \\
0 & 1 & -1
\end{array}\right), \\
H_{s}(r)= & \frac{1}{2}\left[\ell(\ell+1)-\ell^{\prime}\left(\ell^{\prime}+1\right)\right]\left(1+(-1)^{s+\ell+\ell^{\prime}}\right) \\
& \times\left[\xi_{r} \xi_{r}^{\prime}\left(\begin{array}{ccc}
s & \ell & \ell^{\prime} \\
0 & 0 & 0
\end{array}\right)-\xi_{h}^{\prime} \xi_{h} \Omega_{\ell} \Omega_{\ell^{\prime}}\left(\begin{array}{ccc}
s & \ell & \ell^{\prime} \\
0 & 1 & -1
\end{array}\right)\right] \\
& -\xi_{h}^{\prime} \xi_{r}\left(1+(-1)^{s+\ell+\ell^{\prime}}\right) \Omega_{\ell^{\prime}} \Omega_{s}\left(\begin{array}{ccc}
s & \ell & \ell^{\prime} \\
1 & 0 & -1
\end{array}\right) \\
& +\xi_{r}^{\prime} \xi_{h}\left(1+(-1)^{s+\ell+\ell^{\prime}}\right) \Omega_{\ell} \Omega_{s}\left(\begin{array}{ccc}
s & \ell & \ell^{\prime} \\
1 & -1 & 0
\end{array}\right),
\end{aligned}
$$

where $\gamma_{x}=\sqrt{(2 x+1) / 4 \pi}$ and $\Omega_{x}=\sqrt{x(x+1) / 2}$. The $3 \times 2$ arrays are Wigner- $3 j$ symbols that result from the coupling of the angular momenta of the oscillations and the flow involved (Edmonds, 1974). Due to this coupling of angular momenta, the matrix element $H_{n^{\prime} n, \ell^{\prime} \ell}(m)$ is non-vanishing if certain selection rules are fulfilled. The first rule arises from properties of the Wigner- $3 j$ symbols which vanish except when the harmonic degrees $\ell, \ell^{\prime}$, and $s$ satisfy a triangular condition. The second rule follows from Equation (15); the sum of the degrees must be even otherwise the factor $\left(1+(-1)^{s+\ell+\ell^{\prime}}\right)$ in $R_{s}$ and $H_{s}$ vanishes,

$$
s+\ell+\ell^{\prime} \equiv 0 \quad \bmod 2 .
$$



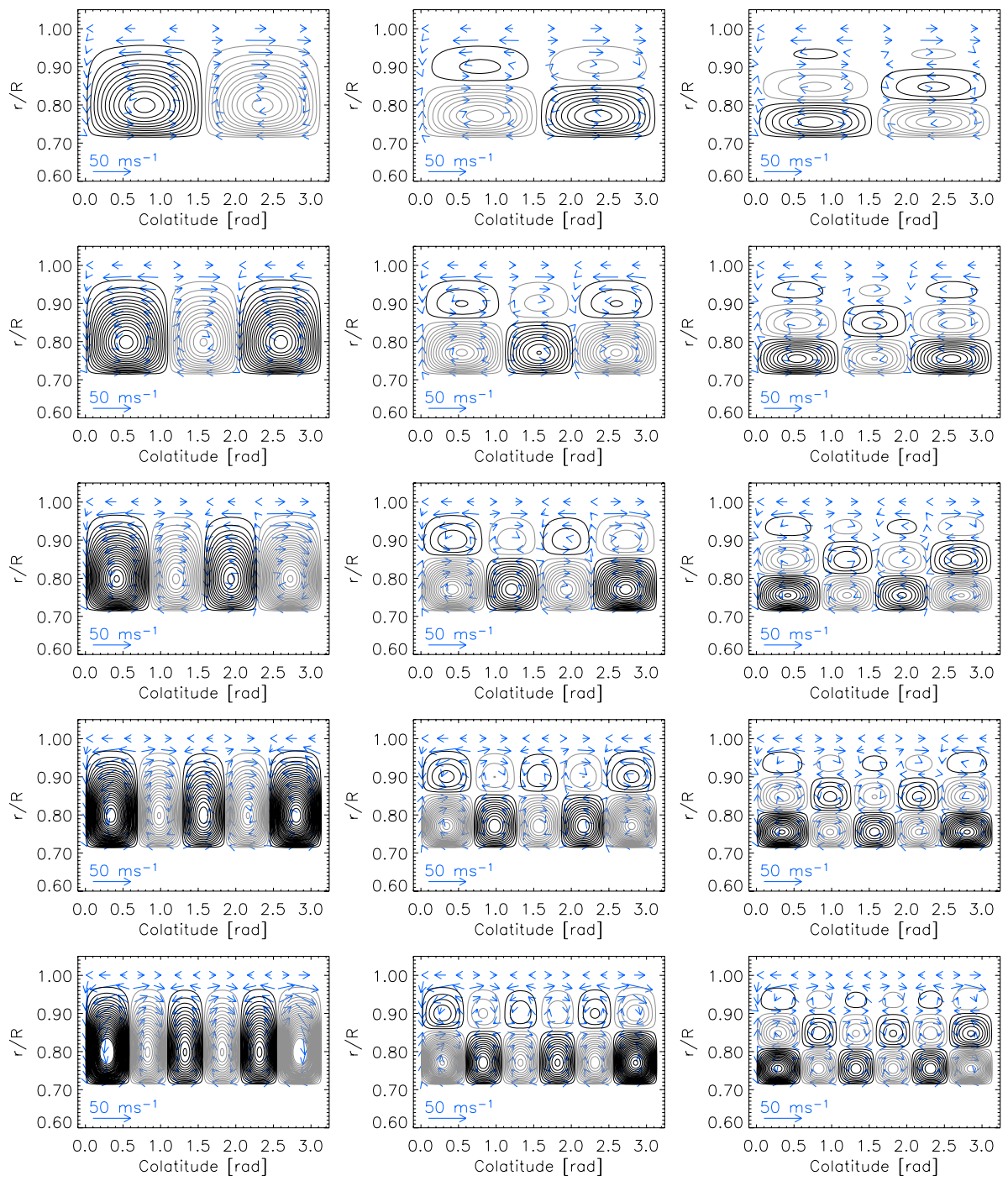

Figure 1 Streamlines for the flows $\rho \boldsymbol{u}_{0}$ of the 15 meridional-circulation models as functions of solar colatitude and radius. Black indicates counterclockwise flow, gray clockwise. The arrows indicate the flow velocity at some positions. The number of cells in horizontal direction changes from top to bottom between two and six. The number of cells in depth changes from left to right between one and three.

We can simplify Equation (14) by defining a poloidal flow kernel $K_{s}(r)$

$$
H_{n^{\prime} n, \ell^{\prime} \ell}(m) \equiv 8 \mathrm{i} \omega_{\mathrm{ref}} \pi(-1)^{m} \gamma_{\ell} \gamma_{\ell^{\prime}} \sum_{s} \gamma_{s} \int_{0}^{R_{\odot}} u_{s} K_{s}(r) \rho_{0} r^{2} \mathrm{~d} r\left(\begin{array}{ccc}
s & \ell & \ell^{\prime} \\
0 & m & -m
\end{array}\right) \text {. }
$$

According to this result, the meridional circulation leads to an effective shift of the eigenvalues, i.e., the squared mode frequencies, if the selection rules are fulfilled. These shifts are given by the eigenvalues $\lambda$ of the matrix $\boldsymbol{Z}$, or in more detail, the corrected mode frequency 


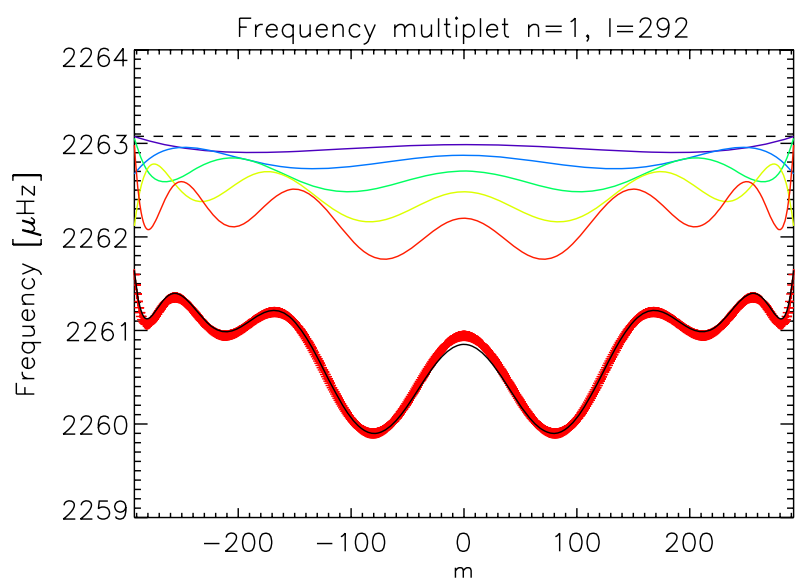

Figure 2 Splittings of the $p$-mode multiplet $n=1, \ell=292, v=2263.08 \mu \mathrm{Hz}$ as a function of azimuthal order $m$. The splittings are caused by different meridional flow models: $s=2, n_{\mathrm{c}}=1$ (purple), $s=3, n_{\mathrm{c}}=1$ (blue), $s=4, n_{\mathrm{c}}=1$ (green), $s=5, n_{\mathrm{c}}=1$ (yellow), $s=6, n_{\mathrm{c}}=1$ (red). The total effect of a sum of these five flow models is displayed by the thick red line, the black line on top of this gives the result for a sum of the five flow models but with $n_{\mathrm{c}}=3$. The unperturbed mode frequency is given by the dashed line.

$\tilde{\omega}_{n l m}$ is given by

$$
\tilde{\omega}_{n l m}^{2}=\omega_{\mathrm{ref}}^{2}+\lambda_{n l m} .
$$

Following the concept of quasi-degenerate perturbation theory, the coupling of only two modes in the presence of only one flow component was investigated by Roth and Stix (1999). They found that due to coupling of two modes by any poloidal velocity field the absolute magnitudes of the frequency shifts are equal for both couplers, but the sign differs: the shift is negative for the mode with the lower frequency. In the case of two multiplets where pairs of modes are coupling, the frequency shifts are a function of the azimuthal order $m$. The functional dependence on $m$ is given by the Wigner-3 $j$ symbol as a function of $m$, and by the factor $(-1)^{m}$. It then follows that the shifts within one multiplet are either always negative or always positive. From this it follows for the case of the meridional circulation that, due to the dependence of the Wigner- $3 j$ symbol on $m$, the frequency shifts are always symmetric about the mode with $m=0$ which in general is also shifted.

We would also like to point out that due to the second selection rule (16), the flow kernel $K_{s}$ vanishes if $\ell^{\prime}=\ell$, i.e. there are no contributions to the diagonal entries on the matrix $\mathbf{Z}$ from the matrix elements $H_{n^{\prime} n, \ell^{\prime} \ell}(m)$. As the matrix elements $H_{n^{\prime} n, \ell^{\prime} \ell}(m)$ are complex and $\mathbf{Z}$ is Hermitian, a change of the orientation of $\boldsymbol{u}_{0}$ does not change the frequency shifts.

\section{Results}

The results of our investigation are frequency shifts for the mode multiplets with $0 \leq \ell \leq 300$ and $1 \leq n \leq 30$ calculated numerically by setting up the full coupling matrix (8), i.e., taking all possible couplings of modes into account and evaluating the eigenvalues.

We first focus on the frequency shifts in the multiplets. According to the properties of the Wigner-3 $j$ symbols, the shifts within a multiplet are symmetric about the mode with $m=0$. Figure 2 displays an example. The frequency splitting is stronger for meridional circulation 

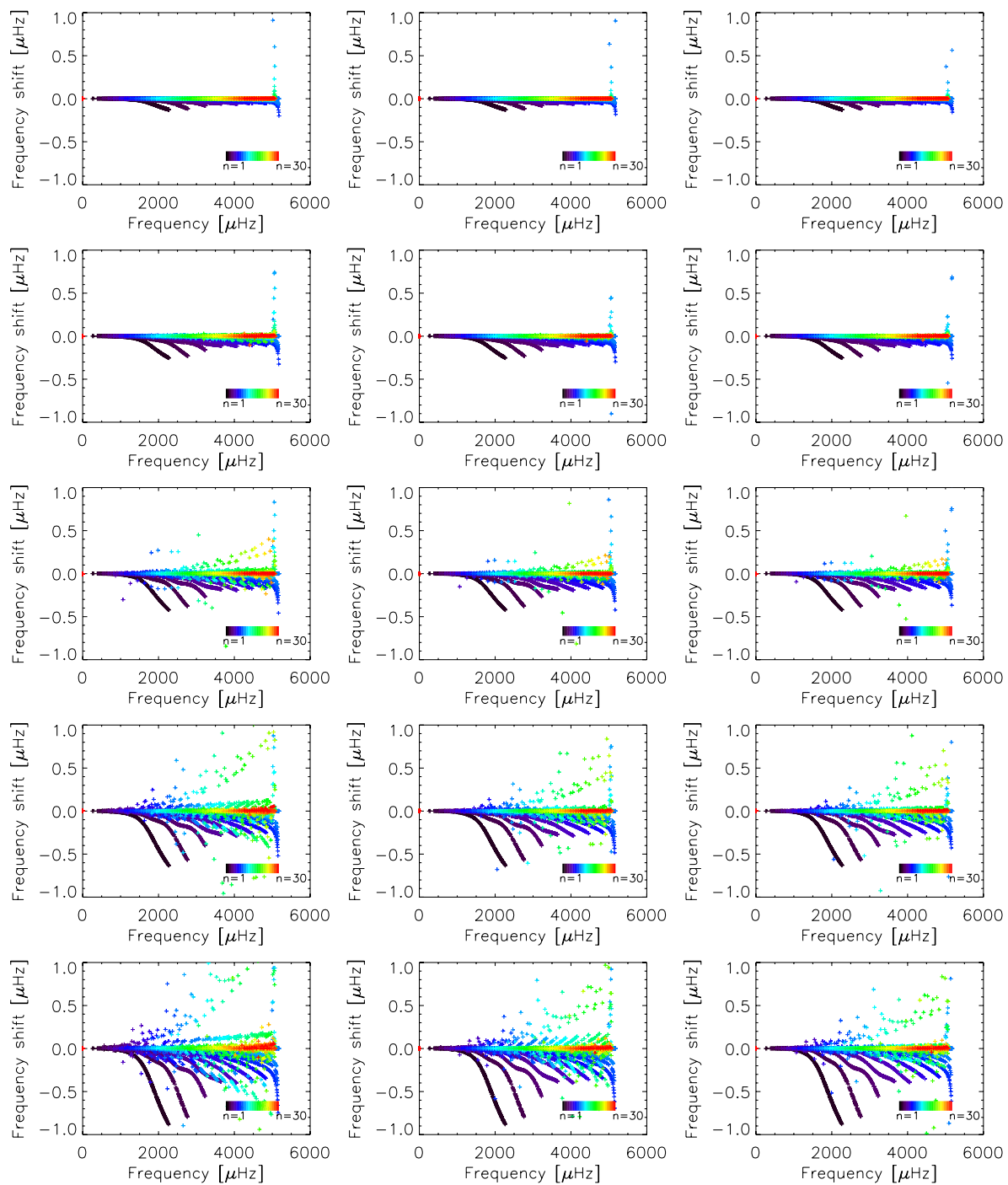

Figure 3 The mean frequency shifts of the multiplets caused by the 15 different configurations of the meridional circulation ( $c f$. Figure 1) as functions of the unperturbed mode frequencies. The color code gives the radial order $(n)$ of the modes. The degree of the meridional circulation affecting the modes changes from $s=2$ (top) to $s=6$ (bottom), the order $n_{\mathrm{c}}$ of the meridional circulation changes from one (left) to three (right).

components with higher degree $s$. In the example of Figure 2 the shifts are on the order of $1 \mu \mathrm{Hz}$ for $s=6$. The overall effect of the superposition of the meridional circulation components results in shifts on the order of $3 \mu \mathrm{Hz}$ away from the unperturbed frequency. The difference between the summed effect of models with $n_{\mathrm{c}}=1$ cell in depth and models with $n_{\mathrm{c}}=3$ cells in depth is on the order of $0.1 \mu \mathrm{Hz}$.

To give a better overview of the effect on all modes, Figure 3 displays the mean frequency shifts of the multiplets as a function of the unperturbed mode frequencies. The frequency 
Figure 4 The frequency difference to the next nearest mode fulfilling the selection rule for the meridional circulation component with $s=2$. The color code gives the radial order of the modes.

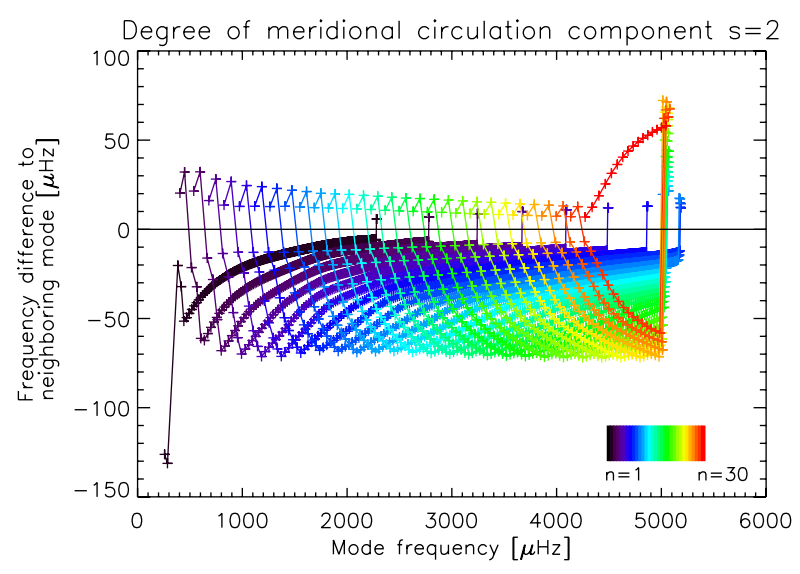

shifts were obtained for the single meridional flow components. One common result is a negative frequency shift of most modes. We find positive frequency shifts only in a few multiplets. The origin of this effect is given by the shape of the $\ell-v$ diagram. According to quasi-degenerate perturbation theory, the multiplet nearest in frequency fulfilling the selection rules causes the strongest shift (Roth and Stix, 1999). As shown for the case $s=2$ in Figure 4, in most cases these nearest neighboring modes have a higher frequency. This is explained by the first selection rule and the curvature of the ridges in the $\ell-v$ diagram. Because of that rule, the difference $\left|\ell-\ell^{\prime}\right|$ of the harmonic degrees of two coupling modes can not be larger than $s$, which is a small number as we consider low degrees $s$ of the flow components. Possible coupling partners for a particular mode can then only come from a very narrow region in $\ell$. As the slopes of the ridges are steeper towards lower harmonic degrees, the frequency spacing between the modes decreases, with increasing $\ell$, along a ridge. In addition, the frequency difference between two ridges increases with $\ell$. Therefore the nearest neighboring mode fulfilling the selection rules usually lies on the same ridge and has a higher frequency and a higher harmonic degree. Figure 5 shows that in most of the investigated cases the coupling partners lie on the same ridge. Interestingly there is some structure visible in these plots. There are areas where all coupling partners are coming from the same ridge or from one ridge above or below. These areas are defined by the curvature of the ridges. With higher degree $s$ of the meridional flow, the coupling of modes from different ridges becomes more frequent. These plots indicate only the position of the nearest coupling partner, because this partner causes the strongest effect. The effects of coupling partners with greater distances in frequency are weaker.

Another common result is that the frequency shifts are strongest for the modes with low radial order $(n)$ and with high harmonic degree $(\ell)$. This is again explained by the location of the nearest neighbor in frequency (Figure 4). The lower the radial order $n$ the smaller the frequency difference to the next mode. Along a ridge, the frequency difference to the next mode decreases causing a stronger splitting of the multiplets. On the ridges with high radial order, the frequency spacing between two neighboring modes is larger than on the ridges with low radial order.

These two common results apply in principle to all poloidal flows, not only to the meridional circulation. Therefore we can conclude that the overall effect of large-scale poloidal flows in the convection zone is a lowering of the mode frequencies. This effect could contribute to reduce the discrepancy between the theoretically calculated mode frequencies and 

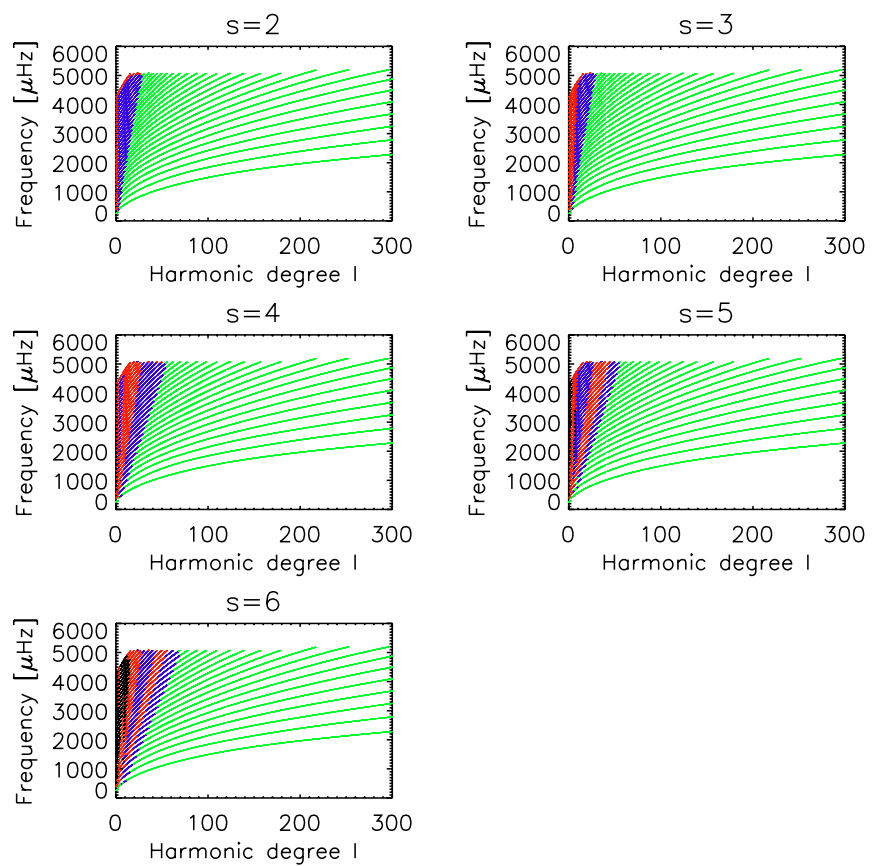

Figure 5 Theoretical $\ell-v$ diagrams with colors indicating the origin of the coupling partner with nearest frequency. Green: coupling partner from the same ridge; blue: coupling partner from one ridge above; red: coupling partner from one ridge below, black: coupling partner is more than one ridge away. The degree $(s)$ of the meridional circulation components changes from two to six.

the observed frequencies. A small frequency reduction has also been found, for a different cause, by Zhugzhda and Stix (1994) and Stix and Zhugzhda (1998).

Investigating the results shown in Figure 3 in detail for the meridional circulation models used, we find that the frequency shifts are increasing from $s=2$ to $s=6$. In the case of $s=2, n_{\mathrm{c}}=1$ the total average of these shifts over all modes affected by the meridional circulation is $0.01 \mu \mathrm{Hz}$, whereas in the case $s=6, n_{\mathrm{c}}=1$ the magnitude of the average shift is $0.1 \mu \mathrm{Hz}$.

There is also a small difference noticeable in the frequency shift if the number $\left(n_{\mathrm{c}}\right)$ of cells changes. The difference of the average shifts calculated for the models $s=2, n_{\mathrm{c}}=1$ and $n_{\mathrm{c}}=3$ is $-0.002 \mu \mathrm{Hz}$. Whereas the difference of the average shifts determined for the models $s=6, n_{\mathrm{c}}=1$ and $n_{\mathrm{c}}=3$ is $-0.03 \mu \mathrm{Hz}$. However, the scatter in the frequency shifts is high. In the case of $s=2, n_{\mathrm{c}}=1$ the largest shift observed is $22.5 \mu \mathrm{Hz}$, and in the case of $s=6, n_{\mathrm{c}}=1$ the largest shift observed is $79.4 \mu \mathrm{Hz}$ (outside the range shown in Figure 3 ). Strong shifts above $10 \mu \mathrm{Hz}$ occur for the modes with high harmonic degree $\ell$.

\section{Discussion}

In this paper we used simple models of the meridional circulation to investigate their influence on the solar $p$-mode frequencies. The simplest model consisted of one cell per hemisphere and depth with a maximum horizontal flow velocity of $15 \mathrm{~m} \mathrm{~s}^{-1}$ on the solar surface. The most complicated model we used had three cells per hemisphere and three cells in depth 
with a horizontal flow velocity of $15 \mathrm{~m} \mathrm{~s}^{-1}$ at the surface, too. We performed numerical calculations based on quasi-degenerate perturbation theory to obtain the frequency splittings of the solar $p$ modes due to these meridional circulation models. We find that the meridional circulation lifts the degeneracy of the multiplets. For the simplest model the shifts are on average only $0.01 \mu \mathrm{Hz}$ with a few shifts up to several $\mu \mathrm{Hz}$. Models with more cells per hemisphere affect the $p$ modes more. For the model with three cells per hemisphere we find an average shift of $0.1 \mu \mathrm{Hz}$, with many shifts of the order of $1 \mu \mathrm{Hz}$. In most cases the shifts are negative due to the fact that the next neighboring mode in frequency dominates the shifting; due to structure of the $\ell-v$ diagram this nearest neighbor has usually a higher frequency, causing therefore negative shifts.

Comparing models with the same number of cells in latitude but different number of cells in depth, we find only tiny differences in the resulting frequency splittings. In the case of $s=6$, the difference in the frequency splitting is on average $0.03 \mu \mathrm{Hz}$. However a number of differences of the order of $1 \mu \mathrm{Hz}$ can occur.

On the Sun, the meridional circulation probably consists of a superposition of flow components with different numbers of cells in depth and latitude. In contrast to the models used in our work, the amplitudes of these flow components are in reality likely to be very different and highly variable in time. Nevertheless, based on our results we are optimistic that the meridional circulation on the Sun could leave an observable signature in the $p$-mode frequencies. In order to detect the effect, a frequency resolution of at least $0.1 \mu \mathrm{Hz}$ must be achieved. This could be done by a several-month-long time series. In order to be able to distinguish not only the various components of the meridional circulation in the orders $s$ but also in the radial orders $n_{\mathrm{c}}$, several years of data need to be averaged to obtain the required precision in the frequencies. As such long data sets exist it will be worth while to search for this effect in the $p$-mode frequencies.

Compared to the splitting caused by the differential rotation, the effect of meridional circulation is small. But as the effect of the rotational splitting is odd in $m$ it can be separated from the even meridional splitting. However, e.g., asphericities and the magnetic field might cause symmetrical frequency shifts, as well. Therefore, a lot of forward modeling will be necessary before the effect of the meridional circulation can be disentangled from these other effects. In this sense, one possible future extension of our work is the determination of the frequency shifts due to more sophisticated models of the meridional circulation, e.g., from three-dimensional numerical models. This might allow tailoring inversion routines for estimating the meridional circulation in the Sun from global solar oscillation frequencies.

Acknowledgements M.R. acknowledges support from the European Helio- and Asteroseismology Network (HELAS) which is funded by the European Commission's Sixth Framework Programme.

Open Access This article is distributed under the terms of the Creative Commons Attribution Noncommercial License which permits any noncommercial use, distribution, and reproduction in any medium, provided the original author(s) and source are credited.

\section{References}

Basu, S., Antia, H.M.: 1997, Seismic measurement of the depth of the solar convection zone. Mon. Not. Roy. Astron. Soc. 287, $189-198$.

Bjerknes, V.: 1926, Solar hydrodynamics. Astrophys. J. 64, $93-121$.

Brun, A.S., Toomre, J.: 2002, Turbulent convection under the influence of rotation: Sustaining a strong differential rotation. Astrophys. J. 570, 865-885.

Choudhuri, A.R., Schüssler, M., Dikpati, M.: 1995, The solar dynamo with meridional circulation. Astron. Astrophys. 303, L29-L32. 
Christensen-Dalsgaard, J., Berthomieu, G.: 1991, Theory of solar oscillations. In: Cox, A.N., Livingston, W.C., Matthews, M.S. (eds.) Solar Interior and Atmosphere, University of Arizona Press, Tucson, 401 478.

Christensen-Dalsgaard, J., Däppen, W., Ajukov, S.V., Anderson, E.R., Antia, H.M., Basu, S., Baturin, V.A., Berthomieu, G., Chaboyer, B., Chitre, S.M., Cox, A.N., Demarque, P., Donatowicz, J., Dziembowski, W.A., Gabriel, M., Gough, D.O., Guenther, D.B., Guzik, J.A., Harvey, J.W., Hill, F., Houdek, G., Iglesias, C.A., Kosovichev, A.G., Leibacher, J.W., Morel, P., Proffitt, C.R., Provost, J., Reiter, J., Rhodes, E.J., Rogers, F.J., Roxburgh, I.W., Thompson, M.J., Ulrich, R.K.: 1996, The current state of solar modeling. Science 272, 1286-1292.

Dikpati, M., Charbonneau, P.: 1999, A Babcock-Leighton flux transport dynamo with solar-like differential rotation. Astrophys. J. 518, 508-520.

Duvall, T.L. Jr.: 1979, Large-scale solar velocity fields. Solar Phys. 63, 3 - 15.

Edmonds, A.R.: 1974, Angular Momentum in Quantum Mechanics, Princeton University Press, Princeton.

Haber, D.A., Hindman, B.W., Toomre, J., Bogart, R.S., Thompson, M.J., Hill, F.: 2000, Solar shear flows deduced from helioseismic dense-pack samplings of ring diagrams. Solar Phys. 192, 335 - 350.

Haber, D.A., Hindman, B.W., Toomre, J., Bogart, R.S., Larsen, R.M., Hill, F.: 2002, Evolving submerged meridional circulation cells within the upper convection zone revealed by ring-diagram analysis. Astrophys. J. 570, $855-864$.

Hathaway, D.H.: 1996, Doppler measurements of the Sun's meridional flow. Astrophys. J. 460, $1027-1033$.

Hill, F.: 1988, Rings and trumpets - Three-dimensional power spectra of solar oscillations. Astrophys. J. 333, $996-1013$.

Kippenhahn, R.: 1963, Differential rotation in stars with convective envelopes. Astrophys. J. 137, $664-678$.

Komm, R.W., Howard, R.F., Harvey, J.W.: 1993, Meridional flow of small photospheric magnetic features. Solar Phys. 147, $207-223$.

Küker, M., Rüdiger, G.: 2005, Differential rotation on the lower main sequence. Astron. Nachr. 326, 265 268.

Küker, M., Stix, M.: 2001, Differential rotation of the present and the pre-main-sequence Sun. Astron. Astrophys. 366, 668-675.

Lavely, E.M., Ritzwoller, M.H.: 1992, The effect of global-scale, steady-state convection and elasticgravitational asphericities on helioseismic oscillations. Roy. Soc. Lond. Phil. Trans. Ser. A 339, $431-$ 496.

Miesch, M.S., Brun, A.S., Toomre, J.: 2006, Solar differential rotation influenced by latitudinal entropy variations in the tachocline. Astrophys. J. 641, 618-625.

Miesch, M.S., Elliott, J.R., Toomre, J., Clune, T.L., Glatzmaier, G.A., Gilman, P.A.: 2000, Three-dimensional spherical simulations of solar convection. I. Differential rotation and pattern evolution achieved with laminar and turbulent states. Astrophys. J. 532, 593-615.

Nandy, D., Choudhuri, A.R.: 2002, Explaining the latitudinal distribution of sunspots with deep meridional flow. Science 296, 1671 - 1673.

Parker, E.N.: 1955, Hydromagnetic dynamo models. Astrophys. J. 122, 293 - 314.

Rempel, M.: 2006a, Flux-transport dynamos with Lorentz force feedback on differential rotation and meridional flow: Saturation mechanism and torsional oscillations. Astrophys. J. 647, 662-675.

Rempel, M.: 2006b, Transport of toroidal magnetic field by the meridional flow at the base of the solar convection zone. Astrophys. J. 637, $1135-1142$.

Ritzwoller, M.H., Lavely, E.M.: 1991, A unified approach to the helioseismic forward and inverse problems of differential rotation. Astrophys. J. 369, 557-566.

Roberts, P.H., Stix, M.: 1972, $\alpha$-Effect dynamos, by the Bullard-Gellman formalism. Astron. Astrophys. 18, $453-466$.

Roth, M., Stix, M.: 1999, Coupling of solar $p$ modes: Quasi-degenerate perturbation theory. Astron. Astrophys. 351, $1133-1138$.

Roth, M., Howe, R., Komm, R.: 2002, Detectability of large-scale flows in global helioseismic data - A numerical experiment. Astron. Astrophys. 396, $243-253$.

Rüdiger, G.: 1980, Reynolds stresses and differential rotation. I - On recent calculations of zonal fluxes in slowly rotating stars. Geophys. Astrophys. Fluid Dyn. 16, 239-261.

Stix, M.: 2004, The Sun: An Introduction, 2nd edn. Springer, Berlin.

Stix, M., Zhugzhda, Y.D.: 1998, Waves in structured media: Non-radial solar $p$ modes. Astron. Astrophys. 335, 685-690.

Tuominen, J., Tuominen, I., Kyröläinen, J.: 1983, Eleven-year cycle in solar rotation and meridional motions as derived from the positions of sunspot groups. Mon. Not. Roy. Astron. Soc. 205, 691-704.

Unno, W., Osaki, Y., Ando, H., Saio, H., Shibahashi, H.: 1989, Nonradial Oscillations of Stars, 2nd edn. University of Tokyo Press, Tokyo.

Wöhl, H., Brajša, R.: 2001, Meridional motions of stable recurrent sunspot groups. Solar Phys. 198, 57 - 77. 
Woodard, M.F.: 2000, Theoretical signature of solar meridional flow in global seismic data. Solar Phys. 197, 11-20.

Zaatri, A., Komm, R., González Hernández, I., Howe, R., Corbard, T.: 2006, North south asymmetry of zonal and meridional flows determined from ring diagram analysis of GONG++ data. Solar Phys. 236, $227-244$

Zhao, J., Kosovichev, A.G.: 2004, Torsional oscillation, meridional flows, and vorticity inferred in the upper convection zone of the sun by time-distance helioseismology. Astrophys. J. 603, 776-784.

Zhugzhda, Y.D., Stix, M.: 1994, Acoustic waves in structured media and helioseismology. Astron. Astrophys. 291, $310-319$. 